\title{
A contribution to appendage drag extrapolation using computational tools
}

\author{
Adriana Oliva Remolà, Luis Pérez Rojas \& Francisco Pérez Arribas \\ Model Basin Research Group (CEHINAV), Naval Architecture Department (ETSIN) \\ Universidad Politécnica de Madrid (UPM)
}

\begin{abstract}
In this paper, the foundations of the beta method, widely used in todays ship appendage extrapolations, are explored.

The present work pretends to validate the Beta Method using experimental and computational tools. The ship used is a rounded bow tugboat with two significant appendages, namely, a midship protective structure for the propulsion system and a stern keel.

The experimental and computational data was obtained through Towing Tank trials and a RANSE CFD code, respectively.
\end{abstract}

\section{INTRODUCTION}

It was William Froude who introduced the concept of scale effect on the appendage drag. His investigations proved that the appendage drag coefficient was smaller for a ship than for its corresponding model, Holtrop (2001). The initial proposed solution, known as the beta method and still commonly applied today, is to reduce the calculated appendage drag coefficient by a factor, usually 50 percent, before adding it to the scaled hulls resistance coefficient.

Nonetheless several other methods exist. The four main ones, along with their limitations, are exposed in ITTC (1984) and ITTC (1990a).

Focusing now on the beta method, the principal drawback is that the wide array of factors involved in the scaling of the appendage drag coefficient, cannot accurately be taken into account by the simple beta coefficient. Another important issue is that the optimum value of the previously mentioned beta factor depends on the Reynold's number. Usually, a value between 0.4 and 0.7 is chosen, although it can be estimated as $\beta=C_{F s} / C_{F m}$, as studied in ITTC (1990b). In addition, with the new advances in CFD codes, the common assumption made that, at equal speeds, the wave resistance coefficient of the bare hull is the same as that of the appended model, is becoming increasingly questionable, Lap (1957).

The main objective herein is to demonstrate if the beta method is suitable in the case of a tugboat with two important appendages. Following the discussion in ITTC (2011), i.e. "computational fluid dynamics may yet be the best way to estimate appendage drag for complicated appendage configuration”,
CFD were chosen as an adequate extrapolated data validation tool of the beta method. When comparing and validate computational simulations, experimental results from the ETSIN Towing Tank trials were used. Similar works and procedures could be found in Obreja et al. (2006).

In the present case, StarCCM+ based on Reynold's Average Navier Stokes Equation (RANSE) was used. With these equations and in order to avoid the complications seen in Pacuraru \& Obreja (2007), a viscous flow was chosen allowing for a numerical estimation of the appendages viscous drag coefficient and its corresponding influence in the total drag. With the intention of properly validating numerical results, the interface air-water was simulated although the free surface can be eliminated if the focus of the project is to find the contribution of the appendage drag, Jiang (2009).

Although experimental results of the drag coefficient of the appended and bare hulls were obtained for a range of velocities (from 8 to 15 knots), in the numerical simulations and their corresponding outputs only a velocity of 12 knots was considered. With these, the relevancy of the beta method is discussed.

\section{EXPERIMENTAL METHOD AND MEASUREMENTS}

The experimental tests were performed at the ETSIN Towing Tank, which has the following dimensions: $100 \mathrm{~m}$ length, $3.8 \mathrm{~m}$ beam and $2.2 \mathrm{~m}$ depth. 


\subsection{Model Construction}

The main dimensions for the model (as the Figure 1) were limited by the installations to the towing tank dimensions and to ITTC requests but also trying to build the model as large as possible to minimize the scale effects.

With the scale factor to be determined first and considering that a larger scale means a reduce influence of the scale factor, a compromise with the ITTC guidelines and the facilities dimensions was reached with a factor of 25 .

The materials used were NECURON 400 for hull and appendages and NECURON 1150 for struts.

Turbulence simulators, $2 \mathrm{~mm}$ tall cylinders with a diameter of $3 \mathrm{~mm}$, were placed at three different places. Namely, along frame $9^{1 / 2}$, on the forward strut at a distance equal to $25 \%$ of its width and on the stern keel at $10 \%$ of its leading edge.

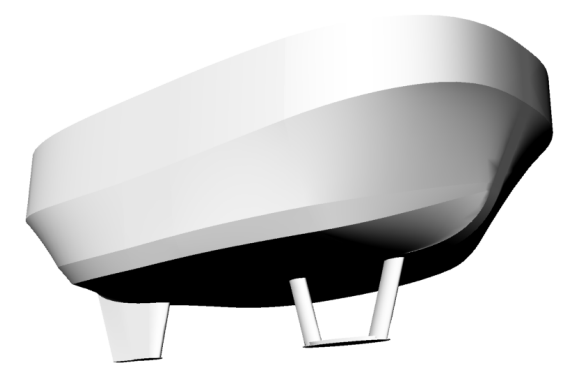

Figure 1: Tugboat model

\subsection{Extrapolation method}

Following the procedure for the beta method, two situations were tested, firstly, trials were carried out without appendages (Case 1), and, in the second Case trials were carried out with the appendages(Case 2). The main parameters for these tests are shown in Table 1.

Table 1: Main parameters of experimental test cases.

\begin{tabular}{lrr}
\hline & Case 1 & Case 2 \\
\hline$L O A(\mathrm{~cm})$ & 106.4 & 106.4 \\
$B_{\text {max }}(\mathrm{cm})$ & 54.0 & 54.0 \\
$T_{\text {bow }}(\mathrm{cm})$ & 12.4 & 12.4 \\
$T_{\text {stern }}(\mathrm{cm})$ & 12.9 & 12.9 \\
$T_{\text {medium }}(\mathrm{cm})$ & 12.7 & 12.7 \\
$S_{W}\left(\mathrm{dm}^{2}\right)$ & 65.3 & 103.3 \\
$k_{\text {Prohaska }}$ & 0.774 & 1.528 \\
$k_{\text {Froude }}$ & 1 & 1 \\
\hline
\end{tabular}

The method used to extrapolate the resistance from the experimental results of the towing tank trials, was the ITTC-78 method. Applying the hypothesis that the form factor is independent of the scale and velocity, the viscous and residual resistance coefficients of the ship can be calculated form the resistance tests. The formula used is as follows:

$C_{t s_{1}}=(1+k) C_{f s}+C_{w}+C_{a}+C_{a a}$
The $\mathrm{C}_{a}$ and $\mathrm{C}_{a a}$ where obtained with the expressions recommended by the ITTC-78. Following with their recommendations and due to the fact that the expression obtained was for ships with a greater length, only half of the calculated $\mathrm{C}_{a}$ value was used. With this, the corrected values used from here onwards:

$$
\begin{aligned}
& C_{a}=6.2 \times 10^{-4} \\
& C_{a a}=6.5 \times 10^{-5}
\end{aligned}
$$

Varying the Froude number between 0.12 and 0.20 , the form factor $k$ for both Cases were initially calculated using the Prohaska method. But, once the resistance were extrapolated, using these form factors (see Table 1), the resistance with appendages was less than without them. To correct this incongruity and with the intention of being conservative, the $k$ value for both Cases were modified to 1 . The reason behind the great disparity found with the procedure used, is that ITTC does not recommended its use in cases where flow separation does exist.

In accordance with the beta method, the expression hereafter was used:

$$
C_{t s_{2}}=C_{t s_{1}}+\beta\left(C_{t m_{2}}-C_{t m_{1}}\right)
$$

Where the $\beta$ factor used was 0.5 .

The sub-indexes (1) and (2) indicate without and with appendages, respectively.

\subsection{Experimental and extrapolated results}

The trial and the extrapolated results (up to now super-index $(*)$ indicate full size Cases), for the velocity of 12 knots for all Cases, are shown in Table 2.

Table 2: Experimental and extrapolated data.

\begin{tabular}{lr}
\hline & Drag Force $(\mathrm{kg})$ \\
\hline Case 1 & 0.988 \\
Case $1 *$ & 14291 \\
Case 2 & 1.223 \\
Case 2* & 15347 \\
\hline
\end{tabular}

For the Case 2, two turbulence configurations for the appendages were considered, with and without turbulence stimulators. The difference between the mentioned configurations is around $1 \%$, hence the values without stimulators will not be considered.

The fluid properties for the trials are exposed in Table 3.

Table 3: Density and viscosity values.

\begin{tabular}{lrr}
\hline & $\rho\left(\mathrm{kg} / \mathrm{m}^{3}\right)$ & $\nu \times 10^{-6}\left(\mathrm{~m}^{2} / \mathrm{s}\right)$ \\
\hline Fresh water & 999.541 & 1.169 \\
Salt water & 1026.126 & 1.184 \\
\hline
\end{tabular}




\section{NUMERICAL CALCULATION METHOD} AND RESULTS

The CFD used was a commercial viscous code based on a finite volume discretization, Star-ccm+, which applies the Reynolds Average Navier-Stokes equation (RANSE) to resolve fluid dynamics problems. The procedure followed was based on, Izquierdo \& González (2010) and Priego et al. (2007).

\subsection{Geometry}

Using Rhinoceros to generate the CAD files, the hull was positioned in its dynamic trim configuration. Due to the symmetry of the problem, only the port side of the hull was meshed, significantly reducing the number of grid cells.

The origin of the reference system is placed in the intersection of the bow with the free surface. The $\mathrm{X}, \mathrm{Y}$ and $\mathrm{Z}$ axes are positive from bow to stern, from symmetry plane to port and positive upward respectively.

With the length overall (LOA) of the model being $1.064 \mathrm{~m}$, as show in Table 1, the computational domain is four LOA lengthwise, one in front and two astern of the ship. One and a half LOA in both the upward direction from the free surface and portside from the centreline. Finally, one LOA was considered for the downward direction from the free surface.

\subsection{Grid}

Of the four Cases simulated in Starccm+, different grids were applied between Case 1 and Case 2 (see section 2.2). For the full size simulations without and with appendages the same grid as for Case 1 and 2 were used, respectively.

For the global domain, a base size grid was defined using equation 3 . This base size grid was used in all Cases. Within this previously defined global domain and in order to refine the meshing of the free surface, several similar control volumes, defined in Table 4 and 5 and showed in Figure 2 , were used for all Cases.

$B S=\frac{1.5 L O A}{5}$

Table 4: Dimensions of Control Volumes.

\begin{tabular}{lrrr}
\hline Volume Control & $\mathrm{X}(\mathrm{m})$ & $\mathrm{Y}(\mathrm{m})$ & $\mathrm{Z}(\mathrm{m})$ \\
\hline Water Surface & full & full & 0.16 \\
Bow & 2.13 & 0.27 & 0.16 \\
Water Pattern & enough & enough & 0.16 \\
\hline
\end{tabular}

It is important to realize that the water pattern control volume was created using a particular coordinate system having its origin in the same place as the principal axes but with the $\mathrm{X}^{*}$ and $\mathrm{Y}^{*}$ rotated $36^{\circ}$, as can be seen in Figure 3.

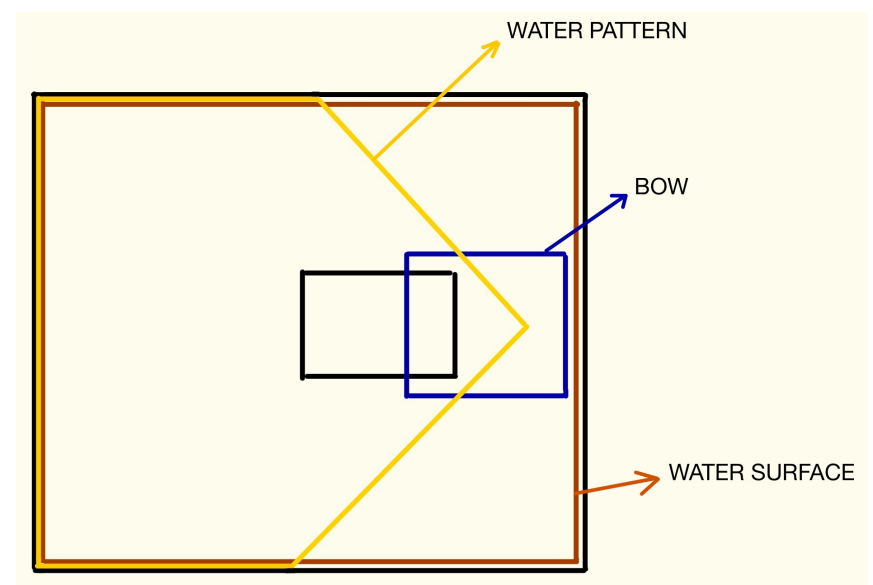

Figure 2: Control Volume Sheme (overhead view)

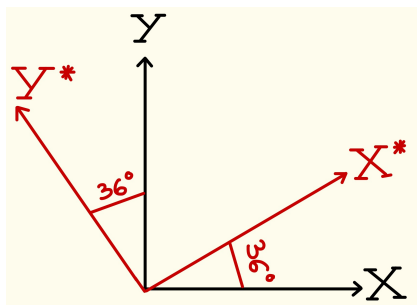

Figure 3: Reference axes rotation, $\mathrm{X}^{*}$ and $\mathrm{Y}^{*}$ (overhead view)

In Case 1, the control volume was meshed anisotropically (according to StarCCM+ parameters) in the $\mathrm{Z}$ direction whilst $\mathrm{X}$ and $\mathrm{Y}$ directions were refined using a percentage of the base size. In Case 2, when following the previously mentioned anisotropic setting for the $\mathrm{Z}$ direction, the interference between appendage and hull could not be properly modelled, thus, resulting in a drag force output of around 15 percent higher than the experimental one, although some appendage refinements were done. An interesting topic left for future work is to mesh Case 1 in the same way.

Table 5: Mesh of Control Volumes.

\begin{tabular}{lcrr}
\hline Volume Control & $\mathrm{X}(\% \mathrm{BS})$ & $\mathrm{Y}(\% \mathrm{BS})$ & $\mathrm{Z}(\% \mathrm{BS})$ \\
\hline & Case 1 & & \\
\hline Water Surface & 50.000 & 50.000 & 13.784 \\
Bow & 25.000 & 25.000 & 6.892 \\
Water Pattern & 12.500 & 12.500 & 3.446 \\
\hline \multicolumn{4}{c}{ Case 2 } \\
Water Surface & 12.500 & 12.500 & 12.500 \\
Bow & 6.250 & 6.250 & 6.250 \\
Water Pattern & 3.125 & 3.125 & 3.125 \\
\hline
\end{tabular}

Figure 4 shows the difference between anisotropic and isotropic mesh near the tugboat's bow.

The surface size of the hull and the prism layer thickness were systematically refined, for all Cases, until the mesh convergence and the Wall $\mathrm{Y}+$ parameter was lesser or equal to 1 . The reason being that, if the value is 1 or below, StarCCM+ assumes that the viscous layer is well resolved and the wall shear stress is computed in a direct numerical simulation. On the downside, the amount of cells required to obtain this value could exceed 
the computers capacity or increase the CPU total simulation time.

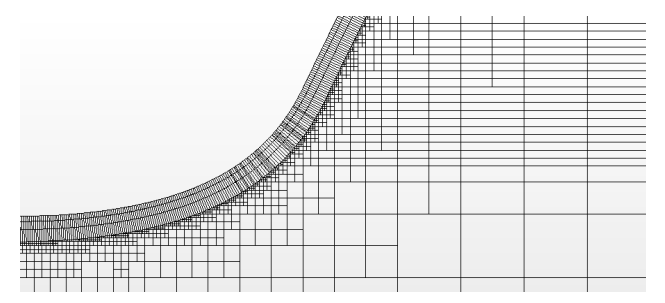

(a) Anisotropic mesh (Case 1)

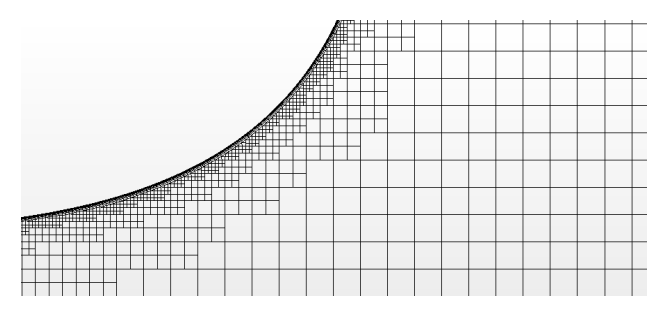

(b) Isotropic mesh (Case 2)

Figure 4: Bow detailed grid (profile view)

Final hull and appendages mesh values are shown in Table 6, where PL represents the Prism Layer.

Table 6: Final hull and appendages mesh values.

\begin{tabular}{lcrr}
\hline \multicolumn{4}{c}{ Case 1 (3.012.017 cells) } \\
\hline & Deck & Hull \\
\hline $\mathrm{N}^{\circ}$ PL & none & 3 & \\
PL Stretch & none & 1.5 & \\
PL Thickness (m) & none & 0.015 & \\
Surface Size (m) & 0.025 & 0.025 \\
\hline \multicolumn{4}{c}{ Case 2 $(8.413 .090$ cells $)$} \\
\hline & Deck & Hull & Append \\
\hline $\mathrm{N}^{\circ}$ PL & none & 8 & 8 \\
$\mathrm{PL}$ stretch & none & 1.5 & 1.5 \\
PL thick (m) & none & 0.015 & 0.015 \\
Surface Size (m) & 0.005 & 0.001 & 0.006 \\
\hline
\end{tabular}

\subsection{Physics}

In order to adequately compare and validate the CFD simulations, two outputs were chosen, namely the drag force and free surface.

From the model Cases drag force, the former was compared and ultimately validated with the experimental data. With the remaining data from the full size Cases, the beta method was evaluated. Leading to results exposed in section 4.

As explained in section 1, the common practice of comparing free surfaces for all simulations, was used. This led to a clear correlation between the simulations of the full size ship and its model, as can be seen in Figure 5.

To model the free-surface flow the Volume Of Fluid wave method (VOF waves) was used. The
VOF waves are applied to simulate surface gravity waves on a light-heavy fluid interface, generating a number of field functions based on wave parameters defined by the user which at the same time define initial and boundary conditions for objects floating in a specified liquid, Hirt \& Nicholson (1981). In the Cases studied, air and water were imposed and a flat wave was created. Current and wind velocities where the parameters specified expressed both as $\left(\mathrm{U}_{0}, 0,0\right)$, being $\mathrm{U}_{0}$ the velocity of the ship.

To solve the motion of a rigid body in response to pressure and shear forces, the fluid exerts the DFBI (Dynamic Fluid Body Interaction) Rotation and Translation module, more information is available in the Starccm+ Manual. Also, no relative moment between the grid domain and the ship was allowed due to the dynamic trim of the boat being previously established in the geometry stage.

The Shear Stress Transport (SST) turbulence model was used. The second order segregated flow and the first order temporal discretization were applied. Also, the all Wall Y+ treatment was enabled.

The no-slip boundary condition was imposed on the solid body as well as on the $\mathrm{Y}_{\max }, \mathrm{Z}_{\max }$ and $\mathrm{Z}_{\min }$ planes. The inflow plane, $\mathrm{X}_{\max }$, was defined with a uniform velocity $\mathrm{U}_{0}$ and the outflow plane, $\mathrm{X}_{\min }$, with the flat VOF wave hydrostatic pressure. The symmetry condition was specified for plane $Y=0$.

The number of iterations used was 5 and the time step was calculated ensuring that the Convective Courant Number was maintained between 1 to 10 .

\subsection{Numerical results}

The drag values and wave profile obtained using numerical tools are showed in Table 7 and Figure 5, respectively.

As a validation of the free surface, wave profiles at $0.5565 B$ with respect to the symmetry plane were compared, adimensionalized by the LOA, see Figure 5.

Table 7: Computational drag values.

\begin{tabular}{lr}
\hline & Drag value $(\mathrm{kg})$ \\
\hline Case 1 & 0.993 \\
Case $1 *$ & 11288 \\
Case 2 & 1.319 \\
Case 2* & 21307 \\
\hline
\end{tabular}

The wave height at $x / L O A=0$ is negative in all Cases, the most probable reason being the existence of a breaking bow wave. Another particularity of the wave profile comparison is that the bare hull and the appended hull present a slightly different wave profile, this is most visible astern of the tugboat. Because of the differences in the control volume grids between Case 1 and Case 2 no final conclusion could be made. Nonetheless, this is most certainly due to the effect of the appendages on the wave profile. 


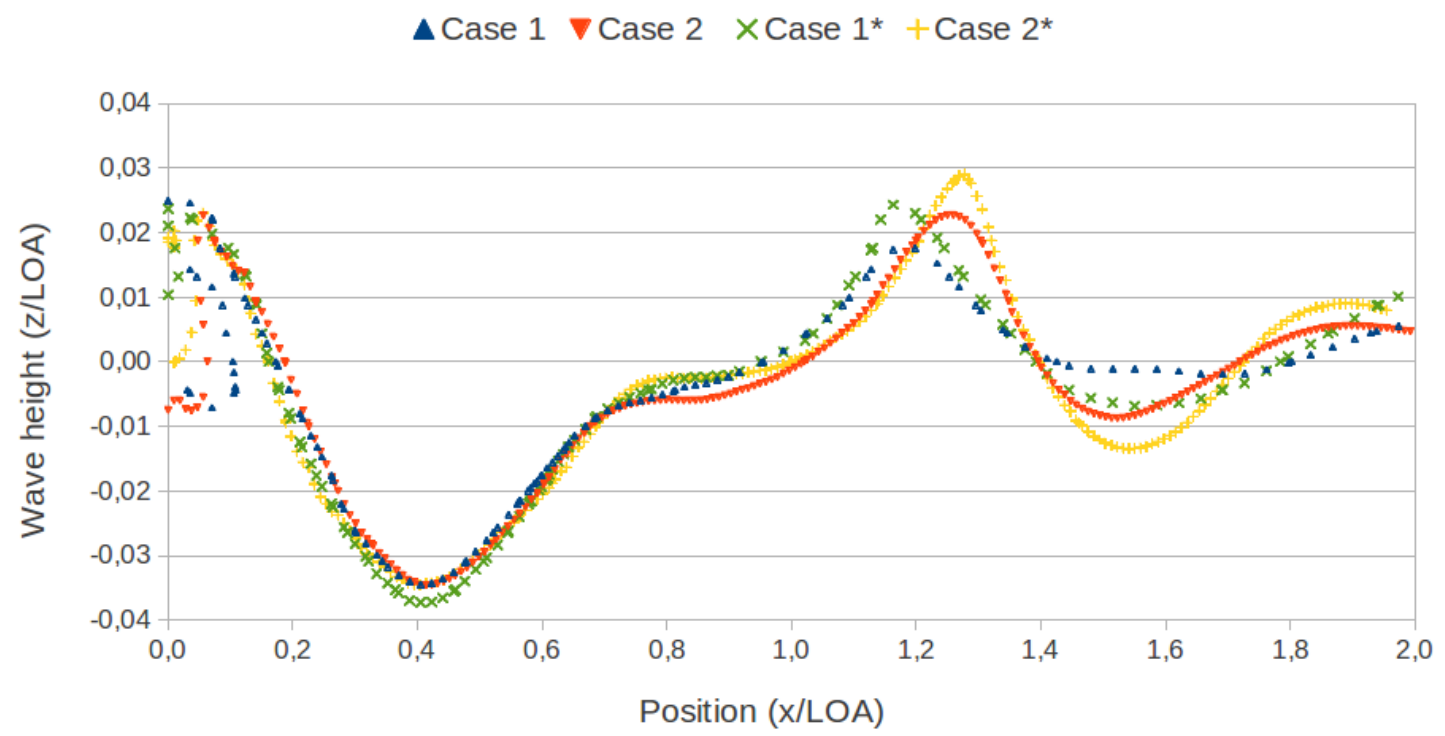

Figure 5: Computational wave profile

In the model simulation Cases, the scale effect on the Reynold's number clearly dampens the wave height, as can be seen in Figure 5, where the differences are greatest for values of $x / L O A \leq 1$. This has been further discussed in Raven et al. (2004) and Raven et al. (2008).

\section{COMPARATIVE ANALYSIS RESULTS}

The drag values obtained for all Cases and their relative differences, expressed as a percentage of the experimental data, are shown in Table 8.

Table 8: Drag values and their relative differences.

\begin{tabular}{lrrr}
\hline \multicolumn{4}{c}{ Model } \\
\hline & Experimental (kg) & Numerical (kg) & $\%$ \\
\hline Case 1 & 0.988 & 0.992 & 0.41 \\
Case 2 & 1.223 & 1.319 & 7.85 \\
\hline \multicolumn{4}{c}{ Full size } \\
\hline \multicolumn{5}{c}{ Extrapolated (kg) } & Numerical (kg) & $\%$ \\
\hline Case 1* & 14291 & 11907 & -16.68 \\
Case 2* & 15347 & 21307 & +38.84 \\
\hline
\end{tabular}

One of the reasons of this discrepancies could be the fact that no turbulence model analysis was carried out, the SST turbulence model is not the most reliable model.

In Case 1,the difference between experimental and numerical data was less than $1 \%$, an acceptable tolerance considering that, although the grid in $\mathrm{Z}$ direction is anisotropic (mentioned in section 3.2), some measurement errors always exist.

In Case $1^{*}$, a pertinent observation shows that the relative difference comes out negative, which could mean that the Froude extrapolation method yields excessively conservative results. However, an improvement in the full size grid could be done in order to obtain more suitable numerical results. The best way would be to refine the meshing of the full sized ship, bringing the present value of the Wall Y+ (approximately 30) down to 1 and ultimately resulting in a StarCCM+ direct calculation of the wall shear stress, as mentioned in section 3.2. With this in mind, the influence that the Reynold's number could have on the turbulence model must not be forgotten.

Focusing on Case 2, the attempts on improving the results obtained, partly through mesh choice, have proven insufficient since the drag difference could only be reduced to $7.85 \%$. This leaves further refinement of the mesh as future work, along with a turbulence model reconsideration.

Finally, in Case $2 *$, the enormous relative drag difference of $+38.84 \%$ indicates that, firstly, the same negative facts to the ones exposed for Case $1^{*}$, are applicable. Secondly, the extrapolation procedures, namely the Beta and Froude method, still convey uncertainties that have an influence on the final extrapolated results.

\section{CONCLUSIONS}

The main objective of the present work was to validate the application of Beta method in a complicated geometry, as a tugboat with significant appendages.

Due to the complex geometry and the existence of flow separation, suitable numerical results could not be obtained. From there, a clear conclusion on the comparison of the computational value (Case $2 *$ ) with the Beta method extrapolated drag could not be reached.

For a conclusive comparison from robust numerical data, future works would be necessary.

Key considerations of said future works would be 
to firstly, refine the grid for Cases $1^{*}$ and $2^{*}$ until a Wall $\mathrm{Y}+$ value lesser or equal to one has been met. Once satisfactory meshes have been obtained, a turbulence model analysis could be carried out for all Cases and their respective meshes. A more rigorous validation of Beta method could be carried out.

Nonetheless, the results and procedure discussed herein still hold some relevance and could be used as a starting point for the validation of appendage drag extrapolation methods.

\section{ACKNOWLEDGEMENTS}

It is an utopia to think that a research work can be done by only one person. At present, any research work needs a team, and the one presented in this paper does not escape from this maxima.

The authors wish to express their sincerely thanks to Hugo Gee who transformed our illegible draft copy into a polished manuscript and to Giorgio Benolli for his contributions during the research information stage.

\section{REFERENCES}

Hirt, C. W. \& B. D. Nicholson (1981). Volume-of-fluid method for dynamics of free boundaries. Joural of Computat Physics 39, 201-210.

Holtrop, J. (2001). Extrapolation of propulsion test for ships with appendages and complex propulsors. Marine Technology, Vol.38, No. 3 38, No. 3, 145-157.

ITTC (1984). Report of the performance comitee. 17th ITTC, 279-282.

ITTC (1990a). Report of the powering performance comitee. 19th ITTC, 255-258.

ITTC (1990b). Report of the powering performance comitee. 19th ITTC, 248-251.

ITTC (2011). Report of the resistance comitee. 26th ITTC, $39-40$.

Izquierdo, J. \& L. M. González (2010). Urans computations of a dtmb 5415. Workshop on CFD in Ship Hydrodynamics.

Jiang, M. (2009). Joint high speed sealift appendage resistance computational fluid dynamics analysis. Naval Surface Warfare Center, Carderock Division.

Lap, A. (1957). Scale effect in the resistance of bossings and shaft brackets. International Shipbuilding Progress 4.

Obreja, D., F. Pacuraru, \& G. Popescu (2006). Investigation of the appendages influence on a tractor tug resistance performance. 2nd Workshop on Vortex Dominated Flows.

Pacuraru, F. \& D. Obreja (2007). Numerical and experimental investigation on a tractor tug resistance performance. 3rd Workshop on Vortex Dominated Flows.

Priego, M., L. M. González, J. Izquierdo, A. Sarasquete, \& L. Pérez Rojas (2007). Ranse with free surface computations around fixed dtmb 5415 model and other baliño's fishing vessels. 9th International Conference on Numerical Ship Hydrodynamics.

Raven, H. C., A. Van der Ploeg, \& B. Strake (2004). Computation of free-surface viscous flows at model and full scale by a steady iterative approach. 25th Symposium on Naval Hydrodynamics.

Raven, H. C., A. Van der Ploeg, B. Strake, \& L. Ea (2008). Toward a cfd-based prediction of ship performance progress in predicting full-scale resistance and scale effects. International Journal of Maritime Engineering. Starccm+. Star-ccm+ Manual. Star-ccm+ 7.04.011. 